Pacific

Journal of

Mathematics

HYPERELLIPTIC LEFSCHETZ FIBRATIONS AND BRANCHED COVERING SPACES

TERRY FULLER 


\title{
HYPERELLIPTIC LEFSCHETZ FIBRATIONS AND BRANCHED COVERING SPACES
}

\author{
TERRY Fuller
}

\begin{abstract}
Let $M$ be a smooth 4-manifold which admits a relatively minimal hyperelliptic genus $h$ Lefschetz fibration over $S^{2}$. If all of the vanishing cycles for this fibration are nonseparating curves, then we show that $M$ is a 2 -fold cover of an $S^{2}$-bundle over $S^{2}$, branched over an embedded surface. If the collection of vanishing cycles for this fibration includes $\sigma$ separating curves, we show that $M$ is the relative minimalization of a Lefschetz fibration constructed as a 2-fold branched cover of $\mathbb{C} P^{2} \#(2 \sigma+1) \overline{\mathbb{C} P^{2}}$, branched over an embedded surface.
\end{abstract}

\section{Introduction.}

As a result of the crucial role currently played by symplectic manifolds in smooth 4-manifold topology, the notion of a smooth Lefschetz fibration has recently taken on renewed importance. It is now known that the existence of such a fibration provides a purely topological description of symplectic 4-manifolds. In particular, by a theorem of Donaldson [D] it is known that every symplectic 4-manifold admits a smooth Lefschetz pencil, which can be blown up to yield a Lefschetz fibration over $S^{2}$. Conversely, Gompf [GS] has shown that every smooth 4-manifold admitting a Lefschetz fibration (with a few understood exceptions) is in fact symplectic, with symplectic fibers.

A genus $h$ Lefschetz fibration (see below for a careful definition) is a smooth fibration $M \rightarrow C$ of an oriented 4-manifold $M$ over an oriented 2manifold $C$ by possibly singular genus $h$ 2-manifolds, where every singular point locally has the holomorphic description of two complex lines meeting in a transverse double point. This strong condition completely determines the local topology of the fibration, with the relevant data phrased in terms of the vanishing cycles of complex algebraic geometry. Intuitively, a vanishing cycle is a simple closed curve on a nonsingular fiber which is gradually shrunk to a point as one approaches the singular fiber. The diffeomorphism classification of regular neighborhoods of possible singular fibers in genus $h$ Lefschetz fibrations is particularly easy to state: Such neighborhoods are determined by whether their corresponding vanishing cycles are nonseparating or separating curves, and in the latter case by the genera of the separated surfaces. 
A natural area of investigation is to study the extent to which an arbitrary smooth Lefschetz fibration globally resembles a holomorphic one. It is conjectured that any hyperelliptic smooth Lefschetz fibration featuring only nonseparating vanishing cycles is holomorphic $[\mathbf{S T}]$. It is known that all holomorphic hyperelliptic Lefschetz fibrations can be obtained as 2-fold covers of ruled surfaces, branched over smoothly embedded curves $[\mathbf{P 1}]$. Our main theorems demonstrate that this feature extends to smooth Lefschetz fibrations over $S^{2}$.

Theorem 4. Let $M \rightarrow S^{2}$ be a relatively minimal hyperelliptic genus $h$ Lefschetz fibration, and assume that all of the vanishing cycles of this fibration are nonseparating curves. Then $M$ is a 2-fold cover of $S^{2} \times S^{2}$ or $S^{2} \widetilde{\times} S^{2}$, branched over an embedded surface.

Theorem 8. Let $M \rightarrow S^{2}$ be a relatively minimal hyperelliptic genus $h$ Lefschetz fibration whose collection of vanishing cycles includes separating curves. Then $M \rightarrow S^{2}$ is the relative minimalization of a Lefschetz fibration $M^{\prime} \rightarrow S^{2}$, and $M^{\prime}$ is a 2-fold cover of a rational surface, branched over an embedded surface. (The terms "relatively minimal" and "relative minimalization" are defined below.)

Since all genus 2 Lefschetz fibrations are hyperelliptic, we immediately have the following corollaries.

Corollary. Let $M \rightarrow S^{2}$ be a relatively minimal genus 2 Lefschetz fibration, and assume that all of the vanishing cycles of this fibration are nonseparating curves. Then $M$ is a 2-fold cover of $S^{2} \times S^{2}$ or $S^{2} \widetilde{\times} S^{2}$, branched over an embedded surface.

Corollary. Let $M \rightarrow S^{2}$ be a relatively minimal genus 2 Lefschetz fibration whose collection of vanishing cycles includes separating curves. Then $M \rightarrow$ $S^{2}$ is the relative minimalization of a Lefschetz fibration $M^{\prime} \rightarrow S^{2}$, and $M^{\prime}$ is a 2-fold cover of a rational surface, branched over an embedded surface.

Our theorems have been proven independently by Siebert and Tian, who demonstrate in addition that the branched coverings of the theorems are branched over symplectically embedded surfaces $[\mathbf{S T}]$. Our corollaries have also been proven independently by Smith $[\mathbf{S}]$.

Following preliminary sections on Lefschetz fibrations and branched coverings, we prove Theorem 4 in Section 3 and Theorem 8 in Section 4. The nature of the proof makes it possible to explicitly describe the branch sets obtained in our theorems, a fact that we will illustrate by example in Section 5.

Of more interest, perhaps, than the theorems themselves is the method of proof, which in each case is to fashion the branched cover by hand, beginning with a handlebody description of $M$ inherited from its structure as a hyperelliptic Lefschetz fibration. Our construction in Section 4 is motivated by 
work of Persson, who constructed genus 2 holomorphic Lefschetz fibrations as double covers of ruled surfaces, and masterfully introduced infinitely close triple point singularities into the branch sets to study questions of complex surface geography $[\mathbf{P 1}]$. The proofs given here generalize Persson's work to the smooth category, and to fibrations of any genus. We do this by considering deformations and resolutions of infinitely close $n$-tuple point singularities, which is discussed in Section 6. We demonstrate that resolutions of infinitely close singularities yield Lefschetz fibrations with separating vanishing cycles, a phenomenon which is known by algebraic geometers to hold for holomorphic genus 2 Lefschetz fibrations [P2]. Thus another facet of this work is to provide a purely topological proof of this correspondence for smooth Lefschetz fibrations of any genus. This correspondence has also been observed by Siebert and Tian for Lefschetz fibrations of any genus [ST], and by Smith for genus 2 Lefschetz fibrations [S].

Acknowledgments. In earlier preprint versions of this work, the author claimed versions of Theorem 4 and Theorem 8 describing all (i.e., non-hyperelliptic) Lefschetz fibrations as simple 3 -fold covers. The author would like to thank Bob Gompf and Bernd Siebert for correspondence which brought to light a gap in those arguments. Indeed, recent work of Siebert and Gang Tian demonstrates that such a result is impossible [ST]. The author would also like to thank Ivan Smith and John Etnyre for valuable conversations.

\section{Lefschetz Fibrations.}

Definition. Let $M$ be a compact, oriented smooth 4-manifold, and let $C$ be a compact, oriented 2-manifold. A proper smooth map $f: M \rightarrow C$ is a Lefschetz fibration if

(1) each critical value $x_{1}, \ldots, x_{\mu}$ of $f$ lies in interior $(C)$; and

(2) about each $f^{-1}\left(x_{i}\right)$ and $x_{i}$, there are complex coordinate charts agreeing in orientation with the orientations of $M$ and $C$, respectively, such that locally $f$ can be expressed as $f\left(z_{1}, z_{2}\right)=z_{1}^{2}+z_{2}^{2}$.

It is a consequence of this definition that

$$
\left.f\right|_{f^{-1}\left(C-\left\{x_{1}, \ldots, x_{\mu}\right\}\right)}: f^{-1}\left(C-\left\{x_{1}, \ldots, x_{\mu}\right\}\right) \rightarrow C-\left\{x_{1}, \ldots, x_{\mu}\right\}
$$

is a smooth fiber bundle over $C-\left\{x_{1}, \ldots, x_{\mu}\right\}$ with fiber diffeomorphic to a 2-manifold $\Sigma_{h}$, and so we also refer to $f$ as a genus $h$ Lefschetz fibration. Two genus $h$ Lefschetz fibrations $f: M \rightarrow C$ and $f^{\prime}: M^{\prime} \rightarrow C^{\prime}$ are equivalent if there are diffeomorphisms $\Phi: M \rightarrow M^{\prime}$ and $\phi: C \rightarrow C^{\prime}$ such that $f^{\prime} \Phi=\phi f$. In this paper, we will always have either $C=D^{2}$, or $C=S^{2}$.

Given a Lefschetz fibration $M \rightarrow C$, one may blow up $M$ to produce a Lefschetz fibration $M \# \overline{\mathbb{C} P^{2}} \rightarrow C$. This will have the effect of replacing a point in one fiber with an embedded 2-sphere of self-intersection number 
-1 . If no fiber of $M \rightarrow C$ contains an embedded sphere of square -1 , we say that $M \rightarrow C$ is relatively minimal. If the Lefschetz fibration $M \rightarrow C$ is obtained from a Lefschetz fibration $M^{\prime} \rightarrow C$ by blowing down all spheres of square -1 found in fibers, we say that $M \rightarrow C$ is the relative minimalization of $M^{\prime} \rightarrow C$.

If $f: M \rightarrow S^{2}$ is a smooth genus $h$ Lefschetz fibration, then we can use the Lefschetz fibration to produce a handlebody description of $M$. Let $M_{0}=M-\nu\left(f^{-1}(x)\right)$, where $\nu\left(f^{-1}(x)\right) \cong \Sigma_{h} \times D^{2}$ denotes a regular neighborhood of a nonsingular fiber $f^{-1}(x)$. Then $\left.f\right|_{M_{0}}: M_{0} \rightarrow D^{2}$ is a smooth Lefschetz fibration. We select a regular value $x_{0} \in \operatorname{interior}\left(D^{2}\right)$ of $f$, an identification $f^{-1}\left(x_{0}\right) \cong \Sigma_{h}$, and a collection of $\operatorname{arcs} s_{i}$ in interior $\left(D^{2}\right)$ with each $s_{i}$ connecting $x_{0}$ to $x_{i}$, and otherwise disjoint from the other arcs. We also assume that the critical values are indexed so that the arcs $s_{1}, \ldots, s_{\mu}$ appear in order as we travel counterclockwise in a small circle about $x_{0}$. Let $V_{0}, \ldots, V_{\mu}$ denote a collection of small disjoint open disks with $x_{i} \in V_{i}$ for each $i$.

To build our description of $M$, we observe first that $f^{-1}\left(V_{0}\right) \cong \Sigma_{h} \times D^{2}$, with $\partial V_{0} \cong \Sigma_{h} \times S^{1}$. Enlarging $V_{0}$ to include the critical value $x_{1}$, it can be shown that $f^{-1}\left(V_{0} \cup \nu\left(s_{1}\right) \cup V_{1}\right)$ is diffeomorphic to $\Sigma_{h} \times D^{2}$ with a 2-handle $H_{1}^{2}$ attached along a circle $\gamma_{1}$ contained in a fiber $\Sigma_{h} \times p t . \subset \Sigma_{h} \times S^{1}$. Moreover, condition (2) in the definition of a Lefschetz fibration requires that $H_{1}^{2}$ is attached with a framing -1 relative to the natural framing on $\gamma_{1}$ inherited from the product structure of $\partial V_{0}$. (See $[\mathbf{G S}]$ and $[\mathbf{K}]$ for proofs of these non-trivial statements, and for more on the topology of Lefschetz fibrations.) For intuition, one should picture the singular fiber $f^{-1}\left(x_{1}\right)$ as being obtained by gradually shrinking the circle $\gamma_{1}$ to a point using the disk obtained from the core of $H_{1}^{2}$; the circle $\gamma_{1}$ is traditionally dubbed a vanishing cycle. In addition, $\partial\left(\Sigma_{h} \times D^{2} \cup H_{1}^{2}\right)$ is diffeomorphic to a $\Sigma_{h}$-bundle over $S^{1}$ whose monodromy is given by $D_{\gamma_{1}}$, a righthanded Dehn twist about $\gamma_{1}$.

Continuing counterclockwise about $x_{0}$, we add the remaining critical values to our description, yielding that

$$
M_{0} \cong f^{-1}\left(V_{0} \cup \bigcup_{i=1}^{\mu} \nu\left(s_{i}\right) \cup \bigcup_{i=1}^{\mu} V_{i}\right)
$$

is diffeomorphic to $\Sigma_{h} \times D^{2} \cup \bigcup_{i=1}^{\mu} H_{i}^{2}$, where each $H_{i}^{2}$ is a 2-handle attached along a vanishing cycle $\gamma_{i}$ in a $\Sigma_{h}$-fiber in $\Sigma_{h} \times S^{1}$ with relative framing -1 . Furthermore,

$$
\partial M_{0} \cong \partial\left(\Sigma_{h} \times D^{2} \cup \bigcup_{i=1}^{\mu} H_{i}^{2}\right)
$$

is a $\Sigma_{h}$-bundle over $S^{1}$ with monodromy given by the product $D_{\gamma_{1}} \cdots D_{\gamma_{\mu}}$. (This product should be read left to right, thus $D_{\gamma_{1}} \cdots D_{\gamma_{\mu}}=D_{\gamma_{\mu}} \circ \cdots \circ D_{\gamma_{1}}$.) 
Since also $\partial M_{0} \cong \Sigma_{h} \times S^{1}$, the global monodromy $D_{\gamma_{1}} \cdots D_{\gamma_{\mu}}$ is necessarily isotopic to the identity.

Finally, to extend our description of $M_{0}$ to $M$, we reattach a regular neighborhood $\nu\left(f^{-1}(x)\right)$. The total space $M$ can then be described as

$$
M=\Sigma_{h} \times D^{2} \cup \bigcup_{i=1}^{\mu} H_{i}^{2} \cup \Sigma_{h} \times D^{2},
$$

where the final $\Sigma_{h} \times D^{2}$ is attached via a $\Sigma_{h}$-fiber preserving map of the boundary. The result is unique up to equivalence for $h \geq 2[\mathbf{K}]$.

Although the description of the monodromy corresponding to each individual critical value $x_{i}$ as a Dehn twist depends on the choice of arc $s_{i}$, other choices of arcs (and of the central identification $f^{-1}\left(x_{0}\right) \cong \Sigma_{h}$ ) do not change the Lefschetz fibration on $M_{0}$, up to equivalence.

We may also reverse this description to construct Lefschetz fibrations, as follows. Starting with a cyclically ordered collection of curves $\left(\gamma_{1}, \ldots, \gamma_{\mu}\right)$ on $\Sigma_{h}$, with $D_{\gamma_{1}} \cdots D_{\gamma_{\mu}}$ isotopic to the identity, we may construct a smooth 4-manifold $M_{0}$ by forming $\Sigma_{h} \times D^{2} \cup \cup_{i=1}^{\mu} H_{i}^{2}$, where the 2-handles $H_{i}^{2}$ are attached in clockwise order along $\gamma_{i} \subset \Sigma_{h} \times\{p t.\} \subset \Sigma_{h} \times S^{1}=\partial\left(\Sigma_{h} \times D^{2}\right)$, each with framing -1 relative to the product framingon $\Sigma_{h} \times S^{1}$. Since $\partial M_{0}$ is a trivial $\Sigma_{h}$-bundle over $S^{1}$, we may then attach $\Sigma_{h} \times D^{2}$ to $M_{0}$ using a $\Sigma_{h}$-fiber preserving map of their boundaries, producing a genus $h$ Lefschetz fibration over $S^{2}$.

Hyperelliptic Lefschetz fibrations. We say that a homeomorphism $\varphi: \Sigma_{h} \rightarrow \Sigma_{h}$ is symmetric if it commutes with the hyperelliptic involution $\iota: \Sigma_{h} \rightarrow \Sigma_{h}$. We let $\mathcal{H}_{h}$ denote the hyperelliptic mapping class group, namely the subgroup of $\mathcal{M}_{h}$ of mapping classes that commute with the class of $\iota$.

Definition. Let $M \rightarrow S^{2}$ be a genus $h$ Lefschetz fibration whose global monodromy is given by the collection $\left(\gamma_{1}, \ldots, \gamma_{\mu}\right)$ of vanishing cycles. Then $M \rightarrow S^{2}$ is a hyperelliptic Lefschetz fibration if there exists a $\varphi \in \mathcal{M}_{h}$ such that $\varphi \circ D_{\gamma_{i}} \circ \varphi^{-1} \in \mathcal{H}_{h}$ for all $1 \leq i \leq \mu$.

The condition for a Lefschetz fibration to be hyperelliptic is equivalent to the supposition that there is an identification of a regular fiber $f^{-1}\left(x_{0}\right)$ with $\Sigma_{h}$ so that each of the vanishing cycles (up to isotopy) satisfies $\iota\left(\gamma_{i}\right)=\gamma_{i}$. We refer to any curve $\gamma$ in $\Sigma_{h}$ with $\iota(\gamma)=\gamma$ as symmetric.

\section{Branched Covers.}

Let $\pi: \Sigma_{h} \rightarrow S^{2}$ denote the usual 2 -fold cover branched over $2 h+2$ points obtained as the quotient of $\iota$, and let $B$ denote the branch set of $\pi$. An easy Euler characteristic argument gives the following. 
Lemma 1. Let $\gamma \subset \Sigma_{h}$ be an embedded symmetric closed curve, and let $\delta=\pi(\gamma)$. Then either:

(a) $\delta$ is an arc with endpoints on $B$, and otherwise disjoint from $B$, with $\pi \mid \gamma: \gamma \rightarrow \delta$ a 2 -fold branched cover; or

(b) $\delta$ is a simple closed curve disjoint from $B$, with $\pi \mid \gamma: \gamma \rightarrow \delta$ a 2-fold (unbranched) cover.

We note that case (a) in the above Lemma occurs when $\gamma$ is a nonseparating curve on $\Sigma_{h}$, and case (b) occurs when $\gamma$ is separating.

Lemma 2. Let $\gamma_{1}$ and $\gamma_{2}$ be embedded symmetric closed curves on $\Sigma_{h}$, and assume that both separate $\Sigma_{h}$ into surfaces of the same genera. Then there exists a symmetric homeomorphism $\tilde{f}: \Sigma_{h} \rightarrow \Sigma_{h}$ and a homeomorphism $f: S^{2} \rightarrow S^{2}$ such that $\tilde{f}\left(\gamma_{1}\right)=\gamma_{2}$ and the diagram

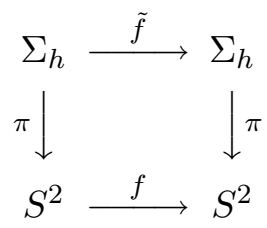

commutes.

Proof. From Lemma 1, case (b), $\pi\left(\gamma_{1}\right)=\delta_{1}$ and $\pi\left(\gamma_{2}\right)=\delta_{2}$ are each simple closed curves in $S^{2}$ which, by assumption, separate $S^{2}$ into regions containing the same number of points of $B$. Thus we may find a homeomorphism $f: S^{2} \rightarrow S^{2}$ fixing the set $B$ with $f\left(\delta_{1}\right)=\delta_{2}$. The mapping class group $\mathcal{M}_{0,2 h+2}$ is known to be generated by "disk twists" about embedded arcs with endpoints on $B$, that is, by homeomorphisms which rotate a small disk neighborhood of the arc by $180^{\circ}$, exchanging the endpoints $[\mathbf{B}]$. Thus we may (after an isotopy) factor $f$ as the product of such disk twists. This product realizing $f$ on $S^{2}$ will lift via $\pi$ to a product $\tilde{f}$ of Dehn twists about symmetric curves, hence to a symmetric homeomorphism with $\tilde{f}\left(\gamma_{1}\right)=\gamma_{2}$.

A separating curve. Let $\gamma_{0}$ be the separating curve on $\Sigma_{h}$ shown in Figure 1, and assume that $\gamma_{0}$ bounds a genus $g$ surface.

Lemma 3. The 4-manifold described by the framed link in Figure 2 is diffeomorphic to $\Sigma_{h} \times D^{2}$. This diffeomorphism maps the dashed curve in Figure 2 to $\gamma_{0} \subset \Sigma_{h} \times\{p t$. $\} \subset \Sigma_{h} \times S^{1}=\partial\left(\Sigma_{h} \times D^{2}\right)$. Furthermore, the product framing on this curve in $\Sigma_{h} \times S^{1}$ agrees with the 0 -framing on this curve (defined by a Seifert surface in $S^{3}$.)

Proof. We will prove the lemma by drawing $\Sigma_{h} \times D^{2}$ as the total space of the 2-fold branched cover $\pi \times i d: \Sigma_{h} \times D^{2} \rightarrow S^{2} \times D^{2}$, using the algorithm of $[\mathbf{A K}]$. Figure 3 shows the base $S^{2} \times D^{2}$ of this cover, with the branch set visible as the $2 h+2$ disjoint horizontal disks $B \times D^{2}$. 


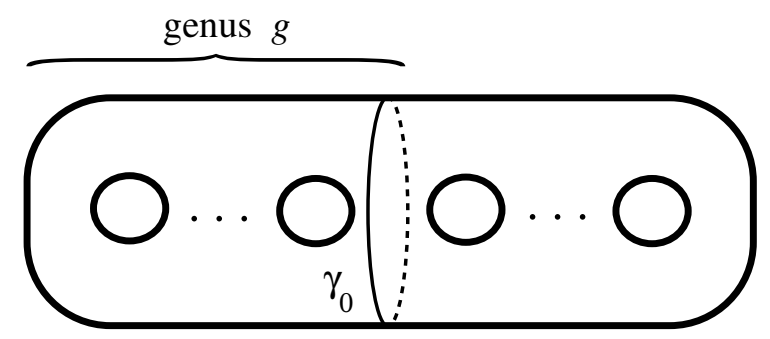

Figure 1.

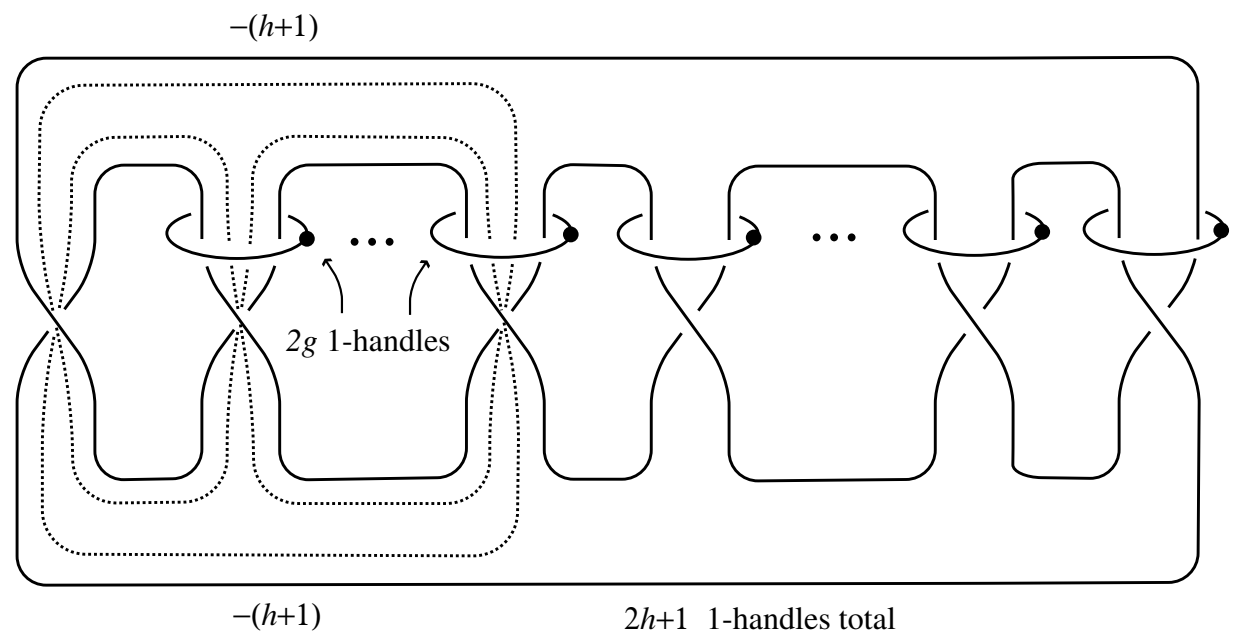

Figure 2.

In this Figure, $\pi \times i d$ restricted to the boundary is easily visualized: Picturing the standard description of the complement of the 0-framed unknot as $D^{2} \times$ $S^{1}$, with the $S^{1}$ factor given by a meridianal circle, we see the $2 h+2$ branch points in each $D^{2} \times\{p t$. $\}$ on the boundary of each meridianal disk. The curve $\gamma_{0}$ is the lift under $\pi$ of the simple closed curve $\delta_{0}$ shown in Figure 4 . The dashed curve in Figure 3 represents $\delta_{0} \subset S^{2} \times\{p t$. $\} \subset S^{2} \times S^{1}=\partial\left(S^{2} \times D^{2}\right)$.

To form the 2-fold branched cover shown in Figure 2, we imagine beginning with the unbranched 2-fold cover given by two disjoint copies of Figure 3. The procedure of $[\mathbf{A K}]$ is to push the interior of the branch set into the interior of $S^{2} \times D^{2}$, cut along the track of this isotopy, and attach handles to glue the copies together. More specifically, gluing the 0-handles of the two copies together along the $2 h+2$ disks of the branch set in Figure 3 


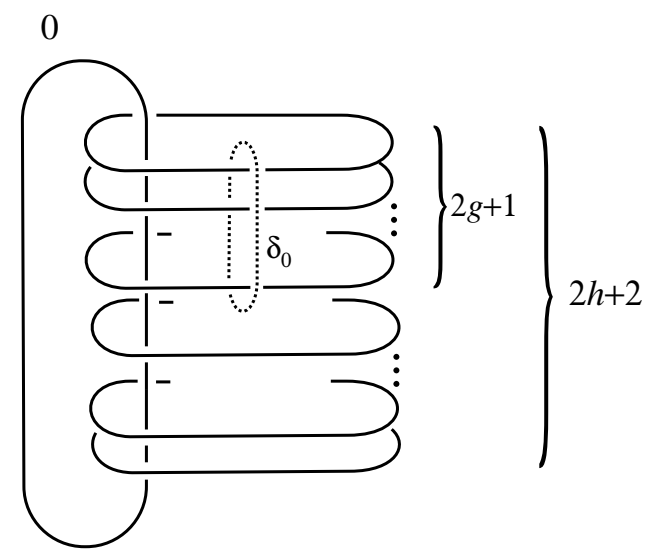

Figure 3.
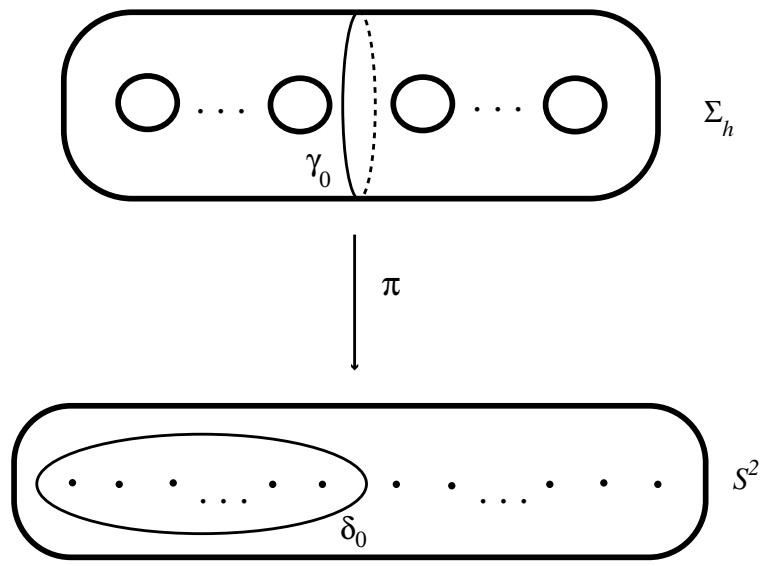

Figure 4.

results in the attachment of $2 h+1$-handles, i.e., the row of dotted circles in Figure 2.

We must also lift the 0-framed 2-handle in Figure 3 to the 2-fold cover. Since its attaching circle intersects the branch set, it will lift to two 2-handles obtained by cutting the attaching circles in each copy of Figure 3 where they intersect the branch set, and joining the endpoints together as we perform the gluings in the previous paragraph. Hence it lifts to the two 2-handles shown in Figure 2. We may calculate the framings of these lifts as in $[\mathbf{A K}]$ : Letting $\lambda_{1}$ and $\lambda_{2}$ denote the 2-dimensional homology classes defined by these lifts of the 2-handle in Figure 3, we must have $\left(\lambda_{1}+\lambda_{2}\right)^{2}=2(0)=0$. 
Expanding, and making use of symmetry, we get $2 \lambda_{1}^{2}+2 \lambda_{1} \cdot \lambda_{2}=0$. Reading $\lambda_{1} \cdot \lambda_{2}=h+1$ directly from Figure 2 , we can then solve $\lambda_{1}^{2}=-(h+1)=\lambda_{2}^{2}$.

Finally, to see that the dashed curve in Figure 2 is $\gamma_{0}$, we simply note that we can draw the lift of $\delta_{0}$ exactly as we lifted the the attaching circles of the 2-handle, with the outcome as pictured. To visualize the product framing on the dashed curve in Figure 2, we can lift a curve parallel to $\delta_{0}$ in a different $S^{2} \times\{p t$. $\}$ fiber. This parallel curve will lift to a curve which lies on the obvious Seifert surface for the dashed curve in Figure 2. This confirms that the product framing and the 0 -framing agree.

\section{Nonseparating Vanishing Cycles.}

We are now ready for our first theorem.

Theorem 4. Let $M \rightarrow S^{2}$ be a hyperelliptic genus $h$ Lefschetz fibration and assume that all of the vanishing cycles of this fibration are nonseparating curves. Then $M$ is a 2-fold cover of $S^{2} \times S^{2}$ or $S^{2} \widetilde{\times} S^{2}$, branched over an embedded surface.

Remarks. It has long been known that every elliptic $(h=1)$ Lefschetz fibration over $S^{2}$ with at least one singular fiber may be obtained as a 2-fold branched cover of $S^{2} \times S^{2}$. This follows from Moishezon's classification of elliptic Lefschetz fibrations [Ms].

It is known that a genus 2 Lefschetz fibration where all vanishing cycles are about nonseparating curves must have $10 n$ singular fibers, for some integer $n \geq 1$ [Ma]. Smith has shown that every genus 2 Lefschetz fibration is a 2 -fold branched cover of $S^{2} \times S^{2}$ when $n$ is even, and is a 2-fold branched cover of $S^{2} \widetilde{\times} S^{2}$ when $n$ is odd [S].

Proof. From Section 1, we can describe $M$ as a handlebody

$$
M=\Sigma_{h} \times D^{2} \cup \bigcup_{i=1}^{\mu} H_{i}^{2} \cup \Sigma_{h} \times D^{2},
$$

where each 2-handle $H_{i}^{2}$ is attached to a symmetric vanishing cycle $\gamma_{i}$ with framing -1 relative to the induced framing coming from $\Sigma_{h} \times S^{1}$. Let $M_{0}$ denote the submanifold $M_{0}=\Sigma_{h} \times D^{2} \cup \bigcup_{i=1}^{\mu} H_{i}^{2} \subset M$. We begin with the 2-fold cover $\pi \times i d: \Sigma_{h} \times D^{2} \rightarrow S^{2} \times D^{2}$ whose branch set consists of the $2 h+2$ disks $B \times D^{2}$ in Figure 3. Our first task is to extend this branched covering to $M_{0}$.

Since each vanishing cycle $\gamma_{i}$ is a nonseparating symmetric curve in $\Sigma_{h} \times$ $\{p t.\} \subset \Sigma_{h} \times S^{1}$, we can apply case (a) of Lemma 1 to each one to produce an arc $\delta_{i} \subset S^{2} \times\{p t$. $\}$. For each $\gamma_{i}$, we then add a band to the branch set of Figure 3 whose core is $\delta_{i}$, and which differs from the band $\delta_{i} \times(p t .-\epsilon, p t$. + $\epsilon) \subset S^{2} \times S^{1}$ by one lefthanded half twist. See Figure 5 . 

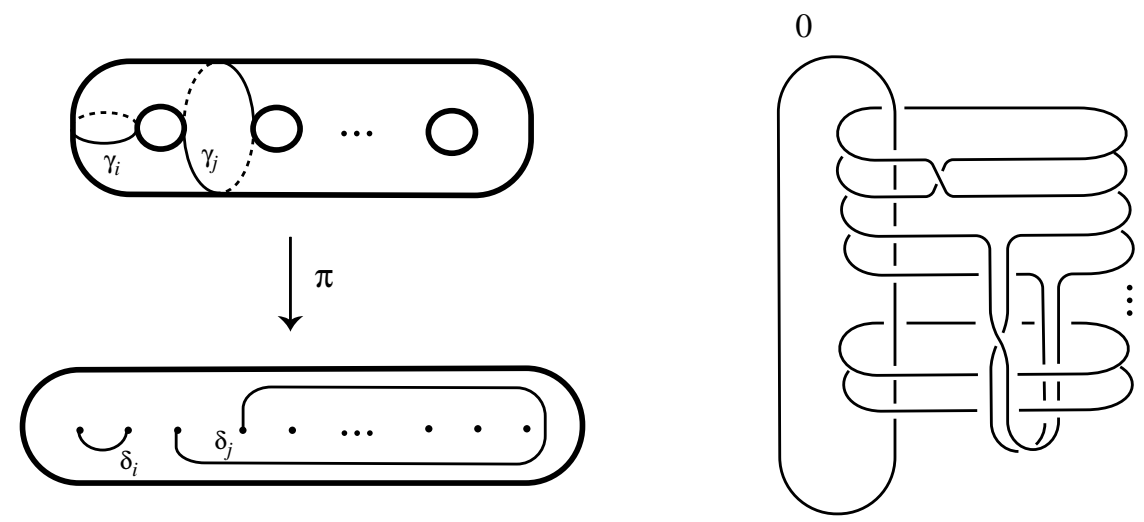

Figure 5 .

This new surface in $S^{2} \times S^{1}$ may contain ribbon singularities, as shown, and following $[\mathbf{M o}]$ we refer to this surface as a ribbon manifold. We may push the interior of this ribbon manifold into the interior of $\Sigma^{2} \times D^{2}$ to produce an embedding.

Proposition 5. The 2-fold cover of $S^{2} \times D^{2}$ branched over the ribbon manifold constructed above (with its interior pushed into the interior of $S^{2} \times D^{2}$ ) is diffeomorphic to $M_{0}$.

Proof of Proposition 5. This follows immediately from the proof of Theorem 6 of [Mo], modified slightly for our setting. For completeness, we summarize the argument and refer the reader to [Mo] for additional details. From Lemma 1, a regular neighborhood $\nu\left(\delta_{i}\right)$ in $S^{2} \times S^{1}$ lifts under $\pi \times i d$ to the regular neighborhood $\nu\left(\gamma_{i}\right)$ in $\Sigma_{h} \times S^{1}$. We attach 2handles $H_{i}^{2}$ to $\Sigma_{h} \times D^{2}$ with attaching circles $\gamma_{i}$ and relative framing -1 via $h_{i}:\left(\partial D^{2} \times D^{2}\right)_{i} \rightarrow \nu\left(\tilde{\gamma}_{i}\right)$. Additionally, we define $g_{i}: H_{i}^{2} \rightarrow H_{i}^{2} / V$ to be the 2-fold cover induced from the involution $V: D^{2} \times D^{2} \rightarrow D^{2} \times D^{2}$ which is reflection in $D^{1} \times D^{1}$. We may assume, after an isotopy, that the involution $h_{i} \circ V \circ h_{i}^{-1}$ on $\nu\left(\gamma_{i}\right)$ agrees with the involution $\iota \times i d$ on $\nu\left(\gamma_{i}\right)$, hence this involution extends over $H_{i}^{2}$. We can then form the map

$$
\pi \times i d \cup \bigcup_{i} g_{i}
$$

from

$$
\Sigma_{g} \times D^{2} \cup \bigcup_{h_{i}} H_{i}^{2}
$$

to

$$
S^{2} \times D^{2} \cup \bigcup_{(\pi \times i d) \circ h_{i} \circ g_{i}^{-1}} H_{i}^{2} / V .
$$

This map is a 2-fold cover whose branch set is isotopic to the ribbon manifold above; the half twist in the bands above are required due to the assumption 
that each $H_{i}^{2}$ is attached with relative framing -1 . However, the addition of the 4-ball $H_{i}^{2} / V$ to the range does not change the manifold, so we have constructed a 2 -fold cover $\Sigma_{h} \times D^{2} \cup \bigcup_{h_{i}} H_{i}^{2} \rightarrow S^{2} \times D^{2}$.

Finally, it remains to extend the branched covering giving $M_{0}$ to all of $M$. From Proposition 5, we have constructed a 2-fold cover $\varphi: M_{0} \rightarrow$ $S^{2} \times D^{2}$. By construction, $\partial M_{0}$ is a $\Sigma_{h}$-bundle over $S^{1}$ with monodromy $H=$ $D_{\gamma_{1}} \cdots D_{\gamma_{\mu}}$, which is isotopic to the identity. Restricted to the boundary, the image of $\varphi$ is an $S^{2}$-bundle over $S^{1}$ whose monodromy is induced from the word $D_{\gamma_{1}} \cdots D_{\gamma_{\mu}}$. Furthermore, $\varphi$ restricted to each fiber of $\partial M_{0}$ is the 2 -fold covering $\pi$. (This may be seen explicitly. The branched covering $\varphi$ projects each Dehn twist $D_{\gamma_{i}}$ to a disk twist about the arc $\delta_{i}$. The boundary of the branch set of $\varphi$ appears as the link $L$ in $S^{2} \times S^{1}$ that is the boundary of the ribbon manifold branch surface in $S^{2} \times D^{2}$. The half twist placed in each band above ensures that $L$ is isotopic to a closed braid. While traversing the $S^{1}$ factor of $S^{2} \times S^{1}$, this braid records the motion of the $2 h+2$ branch points of $\pi$ induced from the monodromy $D_{\gamma_{1}} \cdots D_{\gamma_{\mu}}$.)

We have a $\Sigma_{h}$-bundle equivalence $\Psi: \partial M_{0} \rightarrow \Sigma_{h} \times S^{1}$ obtained by expressing $\partial M_{0}$ as $\Sigma_{h} \times I /(x, 1) \sim(H(x), 0)$, and using the isotopy between $H$ and the identity to adjust the gluing. By $[\mathbf{B H}]$, we may assume that this isotopy is an isotopy through homeomorphisms that preserve the fibers of $\pi$. Thus, if $H_{t}$ denotes this isotopy, then $\pi H_{t}$ projects to an isotopy between $\pi H$ and the identity on $S^{2}$. This gives a bundle equivalence $\psi: S^{2} \times S^{1} \rightarrow S^{2} \times S^{1}$ for which the diagram

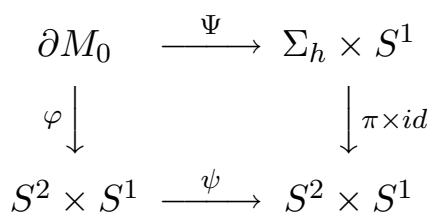

commutes. Therefore,

$$
\varphi \cup(\pi \times i d): M_{0} \cup_{\Psi^{-1}}\left(\Sigma_{g} \times D^{2}\right) \rightarrow\left(S^{2} \times D^{2}\right) \cup_{\psi^{-1}}\left(S^{2} \times D^{2}\right)
$$

describes $M$ as a 2-fold cover of an $S^{2}$-bundle over $S^{2}$, with branch set the closed surface obtained by attaching the disks $B \times D^{2} \subset S^{2} \times D^{2}$ to the ribbon manifold produced in Proposition 5.

\section{Separating Vanishing Cycles.}

A Model Fibration. The construction of the branched covers in the previous section relied crucially on the assumption that all vanishing cycles were nonseparating. In this section, we wish to extend that constuction to achieve arbitrary hyperelliptic Lefschetz fibrations, which may include separating vanishing cycles, as 2 -fold branched covers. To do that, it will be 
necessary to have a local model of how a Lefschetz fibration with a separating vanishing cycle can arise from a branched covering construction.

With this in mind, let $N_{0} \rightarrow D^{2}$ denote the genus $h$ Lefschetz fibration with one singular fiber whose vanishing cycle is the curve $\gamma_{0}$ pictured in Figure 1. We again consider the 2-fold cover $\pi \times i d: \Sigma_{h} \times D^{2} \rightarrow S^{2} \times D^{2}$, whose base is shown in Figure 3. We next would like to extend $\pi \times i d$ to be a branched cover of $S^{2} \times D^{2} \# 2 \overline{\mathbb{C} P^{2}}$. We attach a 2-handle along $\delta_{0} \subset S^{2} \times\{p t$. $\}$ with framing -1 , and we attach another 2-handle along a meridian $\epsilon_{0}$ to $\delta_{0}$ with framing -2 . See Figure 6 .

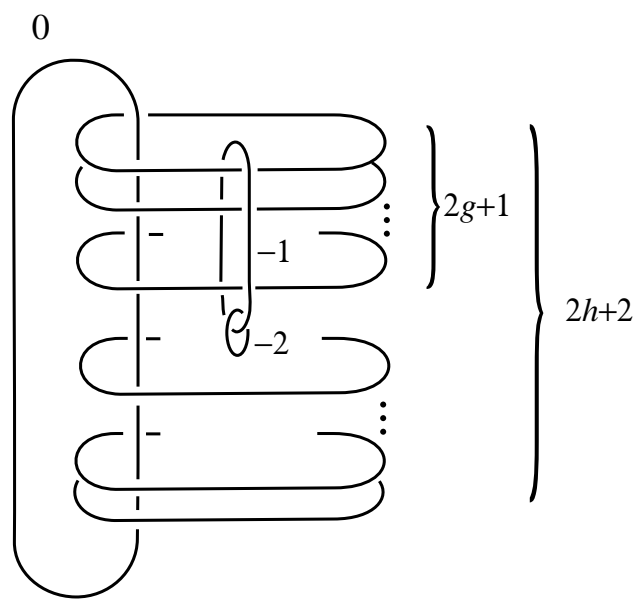

Figure 6.

Blowing down the -1-framed 2-handle shows that this manifold is diffeomorphic to $S^{2} \times D^{2} \# 2 \overline{\mathbb{C} P^{2}}$. We note for future reference that Figure 6 also reveals a boundary diffeomorphism $\partial\left(S^{2} \times D^{2} \# 2 \overline{\mathbb{C} P^{2}}\right) \rightarrow S^{2} \times S^{1}$ obtained by blowing down twice. This boundary diffeomorphism will put two full right-handed twists in the upper strands of the boundary of the branch set. Let $S$ denote the 2 -sphere in $S^{2} \times D^{2} \# 2 \overline{\mathbb{C} P^{2}}$ with $[S]^{2}=-2$ given by the -2-framed 2-handle in Figure 6. To extend $\pi \times i d$, we define $N_{0}^{\prime}$ to be the 2-fold cover of $S^{2} \times D^{2} \# 2 \overline{\mathbb{C} P^{2}}$, branched over $B^{\prime}=B \cup S$.

Proposition 6. There is a non-relatively minimal Lefschetz fibration $N_{0}^{\prime} \rightarrow$ $D^{2}$, and its relative minimalization is $N_{0} \rightarrow D^{2}$. In fact, $N_{0}^{\prime} \cong N_{0} \# \overline{\mathbb{C} P^{2}}$.

Proof. Figure 7 shows a framed link description of $N_{0}^{\prime}$, drawn using the algorithm of $[\mathbf{A K}]$. The branch set $B^{\prime}$ is visible in Figure 6 as $2 h+2$ long horizontal disks union an embedded sphere $S$ of square -2 given by the -2-framed 2-handle. Hence $B^{\prime}$ has a handle decomposition given by 


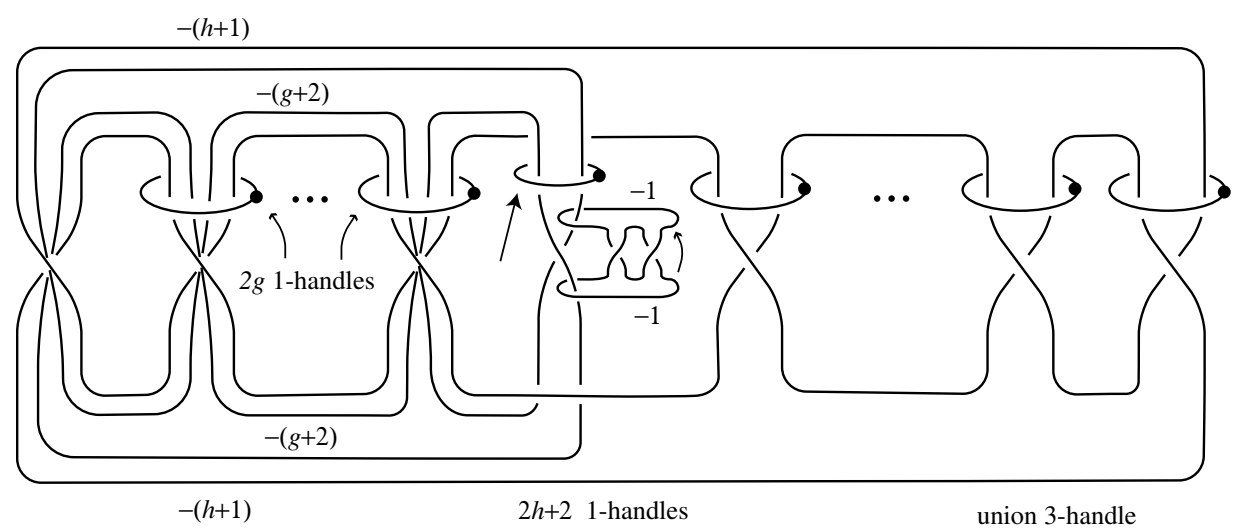

\section{Figure 7.}

$2 h+30$-handles union a 2 -handle (coming from the core of the -2 -framed 2-handle).

To form the 2-fold branched cover shown in Figure 7, we imagine beginning with the unbranched 2-fold cover given by two disjoint copies of Figure 6, and we perform identifications as described in our proof of Lemma 3. Thus, gluing the 0 -handles of the two copies together along the $2 h+2$ disks and the 0-handle of $S$ in Figure 6 results in the row of dotted circles in Figure 7. (The dotted circle marked with an arrow comes from $S$.) As in Lemma 3, the 0-framed 2-handle in Figure 6 lifts to two 2-handles whose framings are $-(h+1)$. The -1 -framed 2 -handle in Figure 6 lifts to the two 2-handles of framing- $(g+2)$, and the -2 -framed 2-handle lifts to the -1 framed 2-handles which link them. Finally, we must glue the two copies of Figure 6 along the 2-handle of $S$. This results in the addition of a 3 -handle to Figure 7, and completes the development of that picture of $N_{0}^{\prime}$.

We next manipulate Figure 7 to see that it has the properties claimed. We cancel the 1-handle marked with an arrow with one of the $-(g+2)$ framed 2-handles. Sliding one of the -1-framed 2-handles over the other as indicated splits off a 0 -framed unknot, which may be moved to be disjoint from the rest of the picture, and used to cancel the 3 -handle. The result is Figure 8. Blowing down the -1 -framed meridian to the -2 -framed 2handle gives Figure 9. Applying Lemma 3, we see that Figure 9 depicts $\Sigma_{h} \times D^{2}$ with 2-handles attached to curves $\gamma_{0}$ and $\alpha$ (where $\alpha$ bounds a disk in $\Sigma_{h} \times\{p t$. $\}$ ), each with relative framing -1 . This is precisely the Lefschetz fibration $N_{0} \rightarrow D^{2}$, blown up at one point.

Examining Figure 9, we recognize the Lefschetz fibration $N_{0} \# \overline{\mathbb{C} P^{2}} \rightarrow D^{2}$ as having two vanishing cycles $\gamma_{0}$ and $\alpha$, where $\alpha$ bounds a disk in $\Sigma_{h} \times\{p t$. $\}$. Analyzing the proof of Proposition 6 more carefully, we have the following. 
$-(h+1)$

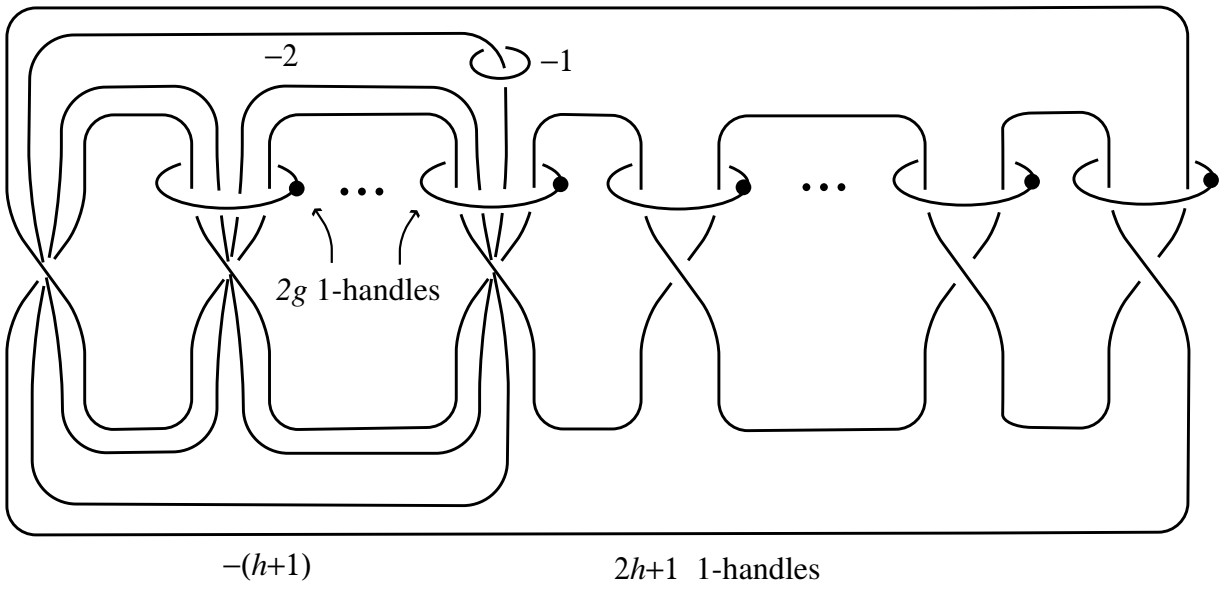

Figure 8.

$-(h+1)$

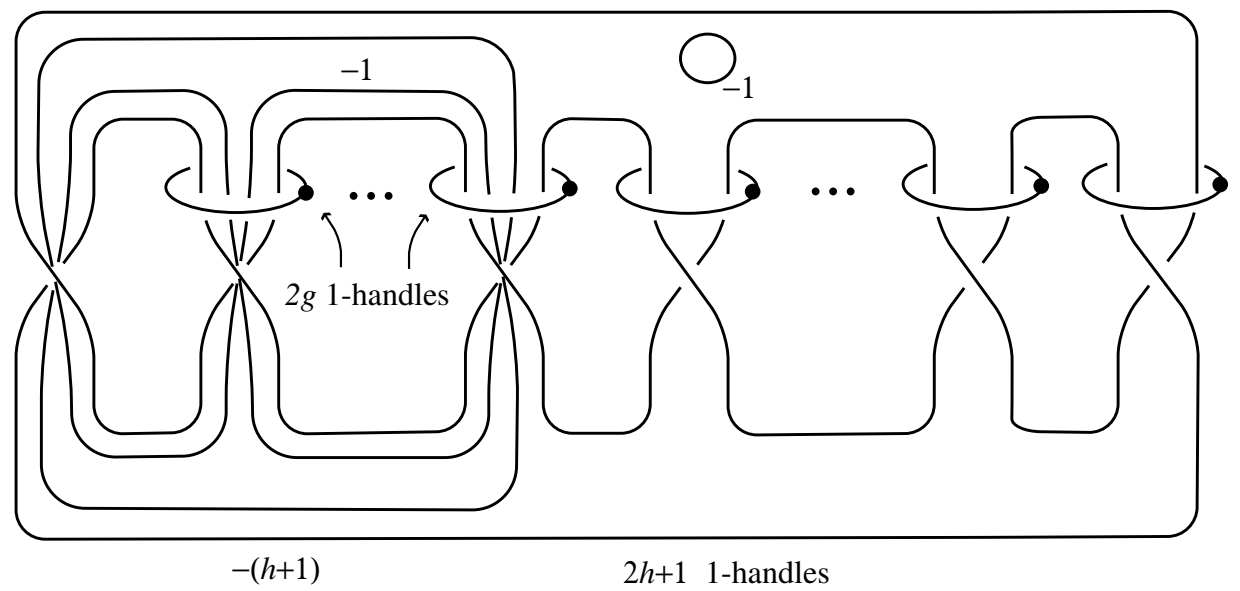

Figure 9.

Corollary 7. Let $\varphi_{0}: N_{0} \# \overline{\mathbb{C} P^{2}} \rightarrow S^{2} \times D^{2} \# 2 \overline{\mathbb{C} P^{2}}$ denote the 2 -fold cover described in the proof of Proposition 6. Then $\varphi_{0}^{-1}\left(\delta_{0}\right)=\gamma_{0}$ and $\varphi_{0}^{-1}\left(\epsilon_{0}\right)=\alpha$.

Proof. We can record $\varphi_{0}^{-1}\left(\delta_{0}\right)$ and $\varphi_{0}^{-1}\left(\epsilon_{0}\right)$ by reenacting the proof of Proposition 6 , recalling that $\delta_{0}$ and $\epsilon_{0}$ are the attaching circles of the -1 - and -2-framed 2-handles, respectively, in Figure 6. Tracking the lifts of these handles through the moves in the above proof gives the corollary. 
Separating Vanishing Cycles. We now state and prove our second main theorem.

Theorem 8. Let $M \rightarrow S^{2}$ be a relatively minimal hyperelliptic genus $h$ Lefschetz fibration whose monodromy includes $\sigma$ separating vanishing cycles. Then $M \rightarrow S^{2}$ is the relative minimalization of a Lefschetz fibration $M \# \sigma \overline{\mathbb{C} P^{2}} \rightarrow S^{2}$, and $M \# \sigma \overline{\mathbb{C} P^{2}}$ is a 2 -fold cover of $\mathbb{C} P^{2} \#(2 \sigma+1) \overline{\mathbb{C} P^{2}}$, branched over an embedded surface.

Proof. From Section 1, we can represent $M$ as a handlebody

$$
M=\Sigma_{h} \times D^{2} \cup \bigcup_{i=1}^{\mu} H_{i}^{2} \cup \Sigma_{h} \times D^{2},
$$

where each 2-handle $H_{i}^{2}$ is attached along a symmetric vanishing cycle $\gamma_{i}$ with framing -1 relative to the induced product framing on $\Sigma_{h} \times S^{1}$. Let $M_{0}=\Sigma_{h} \times D^{2} \cup \bigcup_{i=1}^{\mu} H_{i}^{2} \subset M$. We again begin by forming the 2 -fold cover $\pi \times i d: \Sigma_{h} \times D^{2} \rightarrow S^{2} \times D^{2}$.

We would like to extend $\pi \times i d$ to be a branched covering with $M_{0} \# \sigma \overline{\mathbb{C} P^{2}}$ as its total space. For the 2-handles $H_{i}^{2}$ whose vanishing cycles are nonseparating curves, we may do this as in Section 3. Thus, let $H_{i}^{2}$ be a 2-handle attached along a separating vanishing cycle $\gamma_{i} \subset \Sigma_{h} \times\{p t.\} \subset \Sigma_{h} \times S^{1}$, where $\gamma_{i}$ bounds a genus $g$ surface in $\Sigma_{h} \times\{p t$. $\}$. Let $N_{i}=\Sigma_{h} \times D^{2} \cup H_{i}^{2}$, so that there is a Lefschetz fibration $N_{i} \rightarrow D^{2}$ with one singular fiber whose vanishing cycle is $\gamma_{i}$.

From Lemma 2, we may find a symmetric diffeomorphism $\tilde{f}: \Sigma_{h} \rightarrow \Sigma_{h}$ with $\tilde{f}\left(\gamma_{0}\right)=\gamma_{i}$, and a diffeomorphism $f: S^{2} \rightarrow S^{2}$ such that the following diagram is commutative,

$$
\begin{aligned}
& \left(\Sigma_{h} \times D^{2}, \gamma_{0}\right) \stackrel{\tilde{f} \times i d}{\longrightarrow}\left(\Sigma_{h} \times D^{2}, \gamma_{i}\right) \\
& \pi \times i d \downarrow \downarrow \pi \times i d \\
& \left(S^{2} \times D^{2}, \delta_{0}\right) \stackrel{f \times i d}{\longrightarrow}\left(S^{2} \times D^{2}, \delta_{i}\right)
\end{aligned}
$$

where $\delta_{i}=f\left(\delta_{0}\right)$.

The diffeomorphism $f \times i d: S^{2} \times D^{2} \rightarrow S^{2} \times D^{2}$ along the lower row extends to a diffeomorphism $F: S^{2} \times D^{2} \# 2 \overline{\mathbb{C} P^{2}} \rightarrow S^{2} \times D^{2} \# 2 \overline{\mathbb{C} P^{2}}$, where the range is Figure 3 with a -1-framed 2-handle attached along $\delta_{i} \subset S^{2} \times\{p t$. $\}$ and a -2 -framed 2-handle attached along a meridian to $\delta_{i}$. The diffeomorphism of the upper row extends to a diffeomorphism $\widetilde{F}: N_{0} \rightarrow N_{i}$ (since it takes the vanishing cycle $\gamma_{0}$ to $\gamma_{i}$ ), and further extends (by sending the vanishing cycle $\alpha$ to a vanishing cycle which bounds a disk) to a diffeomorphism $\widetilde{F}: N_{0} \# \overline{\mathbb{C} P^{2}} \rightarrow N_{i} \# \overline{\mathbb{C} P^{2}}$. Finally, we define $\varphi_{i}=F \circ \varphi_{0} \circ \widetilde{F}^{-1}$ : $N_{i} \# \overline{\mathbb{C} P^{2}} \rightarrow S^{2} \times D^{2} \# 2 \overline{\mathbb{C} P^{2}}$, which is a 2 -fold branched cover. This gives a 
commutative diagram

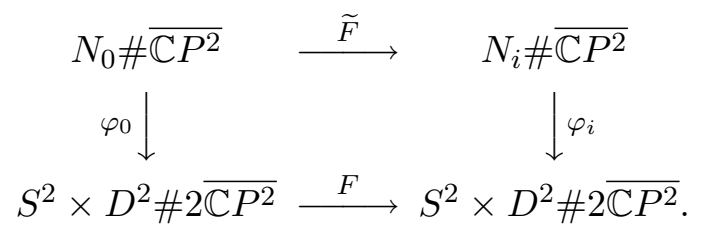

Furthermore, from the commutativity of $(*)$ and Corollary 7 , it follows that the 2 -fold cover $\varphi_{i}: N_{i} \# \overline{\mathbb{C} P^{2}} \rightarrow S^{2} \times D^{2} \# 2 \overline{\mathbb{C} P^{2}}$ may be explicitly described by attaching a -1-framed 2-handle to Figure 3 along $\delta_{i}$, together with a -2 framed 2-handle along a meridian to $\delta_{i}$, and forming the 2-fold cover of $S^{2} \times D^{2} \# 2 \overline{\mathbb{C} P^{2}}$ branched over $B \cup S$. Doing this for all $\sigma$ of the separating vanishing cycles gives a 2 -fold branched covering

$$
\varphi: M_{0} \# \sigma \overline{\mathbb{C} P^{2}} \rightarrow S^{2} \times D^{2} \# 2 \sigma \overline{\mathbb{C} P^{2}} .
$$

Finally, we must extend $\varphi$ to give a branched covering with total space $M \# \sigma \overline{\mathbb{C} P^{2}}$. We can modify the argument from Section 3 to the current situation. Restricting $\varphi$ to the boundary, we have a 2 -fold cover

$$
\partial\left(M_{0} \# \sigma \overline{\mathbb{C} P^{2}}\right) \rightarrow \partial\left(S^{2} \times D^{2} \# 2 \sigma \overline{\mathbb{C} P^{2}}\right) \rightarrow S^{2} \times S^{1},
$$

where the latter map is the boundary diffeomorphism given by blowing down. Let $\omega: \partial\left(M_{0} \# \sigma \overline{\mathbb{C} P^{2}}\right) \rightarrow S^{2} \times S^{1}$ denote this composition.

The boundary $\partial\left(M_{0} \# \sigma \overline{\mathbb{C} P^{2}}\right)$ is a $\Sigma_{h}$-bundle over $S^{1}$ with monodromy $H=D_{\gamma_{1}} \cdots D_{\gamma_{\mu}}$, which is isotopic to the identity. In fact, the monodromy for the bundle on $\partial\left(M_{0} \# \sigma \overline{\mathbb{C} P^{2}}\right)$ has an additional $\sigma$ Dehn twists about curves which bound disks, which are of course isotopic to the identity and can therefore be ignored. The image of $\omega$ is an $S^{2}$-bundle over $S^{1}$ whose monodromy is induced from the word $D_{\gamma_{1}} \cdots D_{\gamma_{\mu}}$. Restricted to each fiber of $\partial\left(M_{0} \# \sigma \overline{\mathbb{C} P^{2}}\right), \omega$ agrees with $\pi$. (As in the strictly nonseparating case of Section 3, we can see the branching explicitly in the boundary. The branched covering $\omega$ now projects each Dehn twist $D_{\gamma_{i}}$ to a disk twist about the $\operatorname{arc} \delta_{i}$ for each nonseparating $\gamma_{i}$, and to $D_{\delta_{i}}^{2}$ for each separating $\gamma_{i}$. The branch set of $\omega$ appears as the link $L$ in $S^{2} \times S^{1}$ that is the boundary of the ribbon manifold branch set corresponding to the nonseparating vanishing cycles, with an additional pair of full right-handed twists corresponding to each separating vanishing cycle. These twists arise from blowing down $\partial\left(S^{2} \times D^{2} \# 2 \sigma \overline{\mathbb{C} P^{2}}\right)$ to $S^{2} \times S^{1}$, as described in Section 3. Thus, as in Section $3, L$ is isotopic to a closed braid, and this braid records the motion of the $2 h+2$ branch points of $\pi$ induced from the monodromy $D_{\gamma_{1}} \cdots D_{\gamma_{\mu}}$.)

We again have a $\Sigma_{h}$-bundle equivalence $\Psi: \partial M_{0} \rightarrow \Sigma_{h} \times S^{1}$ obtained by expressing $\partial M_{0}$ as $\Sigma_{h} \times I /(x, 1) \sim(H(x), 0)$, and using the isotopy between $H$ and the identity to adjust the gluing. Thus, letting $H_{t}$ denote this isotopy, by $[\mathbf{B H}]$ we may assume that $\pi H_{t}$ projects this to an isotopy between $\pi H$ 
and the identity on $S^{2}$. This gives a bundle equivalence $\psi: S^{2} \times S^{1} \rightarrow S^{2} \times S^{1}$ for which the diagram

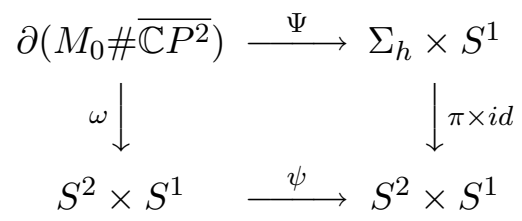

commutes. Therefore,

$\varphi \cup(\pi \times i d):\left(M_{0} \# \sigma \overline{\mathbb{C} P^{2}}\right) \cup_{\Psi^{-1}}\left(\Sigma_{h} \times D^{2}\right) \rightarrow\left(S^{2} \times D^{2} \# 2 \sigma \overline{\mathbb{C} P^{2}}\right) \cup_{\zeta^{-1}}\left(S^{2} \times D^{2}\right)$

describes $M \# \overline{\mathbb{C} P^{2}}$ as a 2 -fold cover of $\left(S^{2}\right.$-bundle over $\left.S^{2}\right) \# 2 \sigma \overline{\mathbb{C} P^{2}}$, where $\zeta$ is the composition of the diffeomorphism $\partial\left(S^{2} \times D^{2} \# 2 \sigma \overline{\mathbb{C} P^{2}}\right) \rightarrow S^{2} \times S^{1}$ and $\psi$. The branch set is the closed surface obtained by attaching the disks $B \times D^{2} \subset S^{2} \times D^{2}$ to $L$ via $\zeta^{-1}$. The proof is completed by noting that $\left(S^{2}\right.$-bundle over $\left.S^{2}\right) \# 2 \sigma \overline{\mathbb{C} P^{2}} \cong \mathbb{C} P^{2} \#(2 \sigma+1) \overline{\mathbb{C} P^{2}}$.

\section{An Example.}

We illustrate Theorems 4 and 8 by describing the branch set in the following example.

In [Ma], Matsumoto showed that $T^{2} \times S^{2} \# 4 \overline{\mathbb{C} P^{2}}$ admits a genus 2 hyperelliptic Lefschetz fibration over $S^{2}$ with eight singular fibers. The global monodromy of this fibration is described by the ordered collection $\left(\beta_{1}, \gamma_{0}, \beta_{2}\right.$, $\left.\beta_{3}\right)^{2}$ of vanishing cycles, where $\beta_{1}, \beta_{2}, \beta_{3}, \gamma_{0}$ are the curves on $\Sigma_{2}$ shown in Figure 10. Let $f=D_{\alpha}$, where $\alpha$ is as shown in Figure 10. We define $M$ to be the genus 2 Lefschetz fibration over $S^{2}$ with global monodromy

$$
\left(\beta_{1}, \gamma_{0}, \beta_{2}, \beta_{3}\right)^{2}\left(f\left(\beta_{1}\right), f\left(\gamma_{0}\right), f\left(\beta_{2}\right), f\left(\beta_{3}\right)\right)^{2} .
$$

(This example was purposely constructed to include a separating curve other than $\gamma_{0}$.)

It is clear that $f$ fixes $\beta_{1}$ and $\beta_{3}$. The curves $\beta_{1}, \beta_{2}, f\left(\beta_{2}\right)$ and $\beta_{3}$ are nonseparating, and are lifts under $\pi: \Sigma_{2} \rightarrow S^{2}$ of the indicated $\operatorname{arcs}$ in $S^{2}$. Thus, each time they occur as a vanishing cycle, they contribute a halftwisted band (relative to the product) to the ribbon manifold branch set, attached according to the corresponding arc. The curves $\gamma_{0}$ and $f\left(\gamma_{0}\right)$ are separating, and are lifts of the indicated simple closed curves in $S^{2}$. Whenever they appear as a vanishing cycle, we attach a -1-framed 2-handle to this curve, together with a -2-framed 2-handle attached to its meridian. Thus we can draw the portion of the branch set residing in $S^{2} \times D^{2} \# 8 \overline{\mathbb{C} P^{2}}$ as in Figure 11. When we close off $S^{2} \times D^{2} \# 8 \overline{\mathbb{C} P^{2}}$ by attaching $S^{2} \times D^{2}$, this branch surface is closed off by six disks attached to the boundary of the ribbon manifold. Forming the 2 -fold branched cover gives a non-relatively 

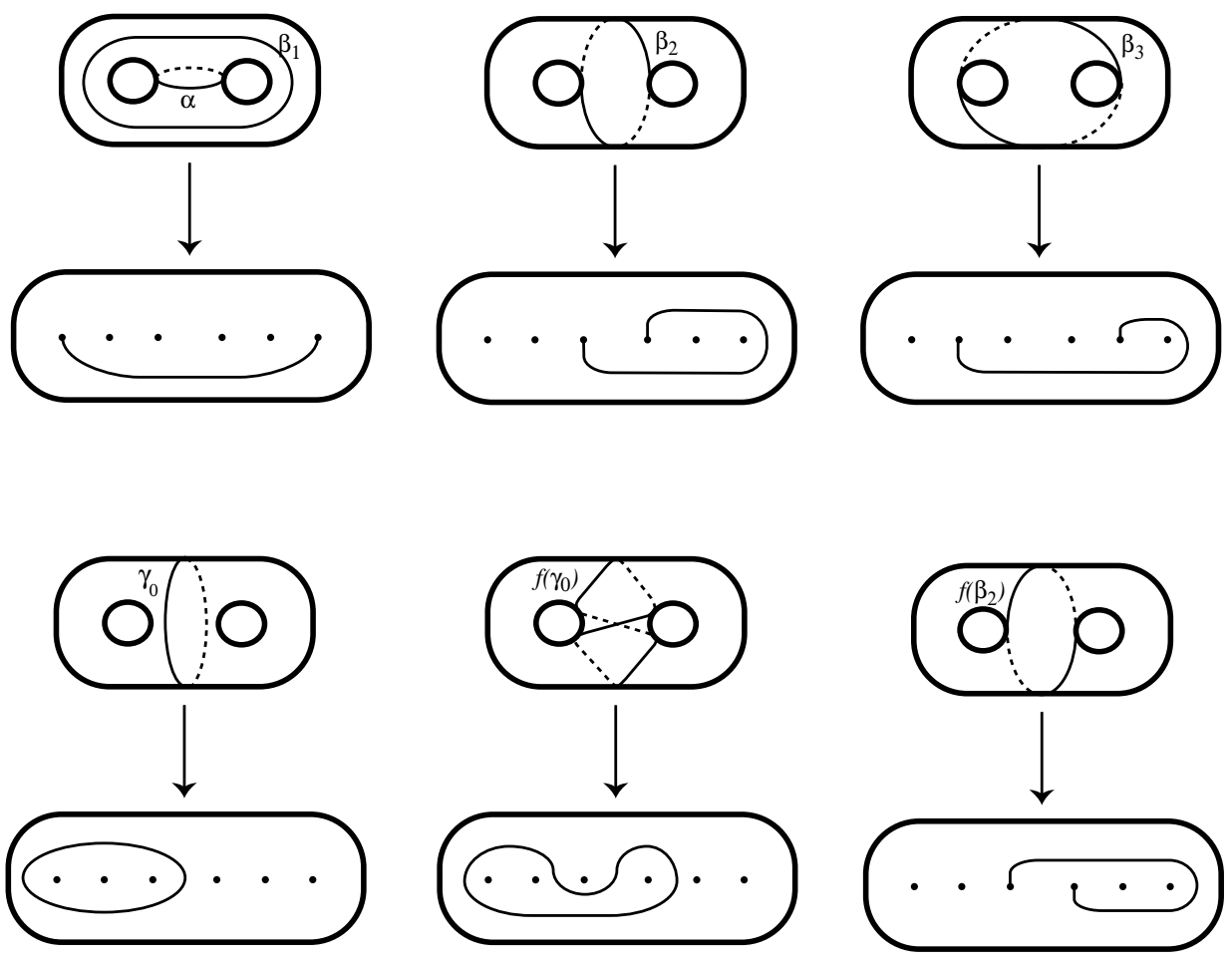

Figure 10.

minimal Lefschetz fibration on $M \# 4 \overline{\mathbb{C} P^{2}}$, which has $M$ as its relative minimalization.

\section{Deformations versus Resolutions of Branch Surfaces.}

In this section, we show that the branch surfaces for the branched covers constructed in this paper are related to basic desingularization processes in complex algebraic geometry.

Let $f \in \mathbb{C}[z, w]$ be a complex polynomial, and assume that $f$ has an isolated singularity at the origin. Let

$$
V=\left\{(z, w) \in \mathbb{C}^{2}: f(z, w)=0\right\} \cap B_{\varepsilon}^{4},
$$

where $B_{\varepsilon}^{4}$ is a 4 -ball centered at the origin of radius $\varepsilon$, with $\varepsilon$ chosen small enough so that $B_{\varepsilon}^{4}$ contains only the singularity at $\mathbf{0}$ and so that $V$ intersects $\partial\left(B_{\varepsilon}^{4}\right)$ transversely. We view $V$ as a local model for a singular portion of a branch surface inside of a 4-manifold, which we would like to desingularize in order to form a smooth branched cover. There are then two standard methods from complex geometry to do this. On one hand, we may deform 


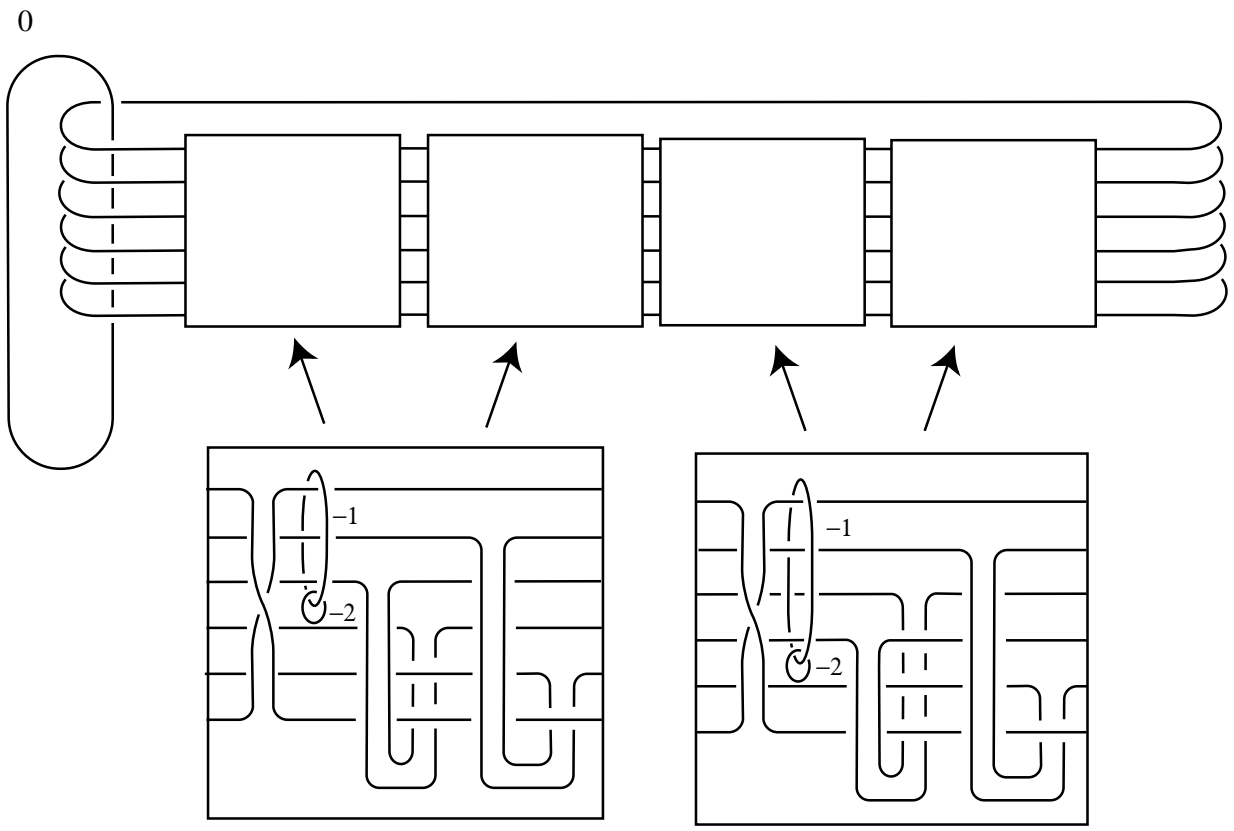

Figure 11.

$V$ into

$$
V_{d}=\left\{(z, w) \in \mathbb{C}^{2}: f(z, w)=t\right\} \cap B_{\varepsilon}^{4},
$$

which will be smooth for $0<|t| \ll \varepsilon$. On another hand, we may form a resolution $V_{r}$ of $V$, which is obtained by repeatedly blowing up $V$ until it becomes smooth. While any $V_{r}$ so produced may not be unique, the minimal resolution of the surface singularity obtained by forming the double cover branched over $V_{r}$ and blowing down any leftover exceptional curves is unique $[\mathbf{B P V}]$. See $[\mathbf{G S}]$ and $[\mathbf{H K K}]$ for further expositions, from a topological perspective, of these processes.

We demonstrate these procedures with a key example.

Example. Let $f(z, w)=z^{n}+w^{2 n}$, where $n=2 g+1$ is odd. Then $V$ is homeomorphic to a cone on an $(n, 2 n)$ torus link. The singular point of $V$ is known in algebraic geometry as an infinitely close $n$-tuple point. We wish to compare $V_{d}$ and $V_{r}$, as well as the 2-fold covers formed with each as a branch set.

The deformation $V_{d}$ of $V$ in $B^{4}$ is known to be isotopic to the fibered Seifert surface of minimal genus in $S^{3}$ of an $(n, 2 n)$ torus link [Mi]. See Figure 12. Figure 13 shows a Kirby calculus picture of the 2-fold cover of $B^{4}$, branched over this surface (with its interior pushed into $B^{4}$ ) $[\mathbf{A K}]$. This 


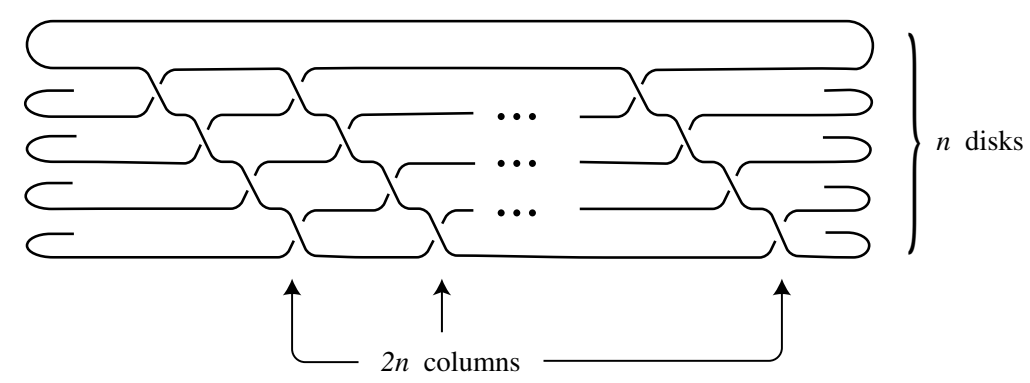

Figure 12.

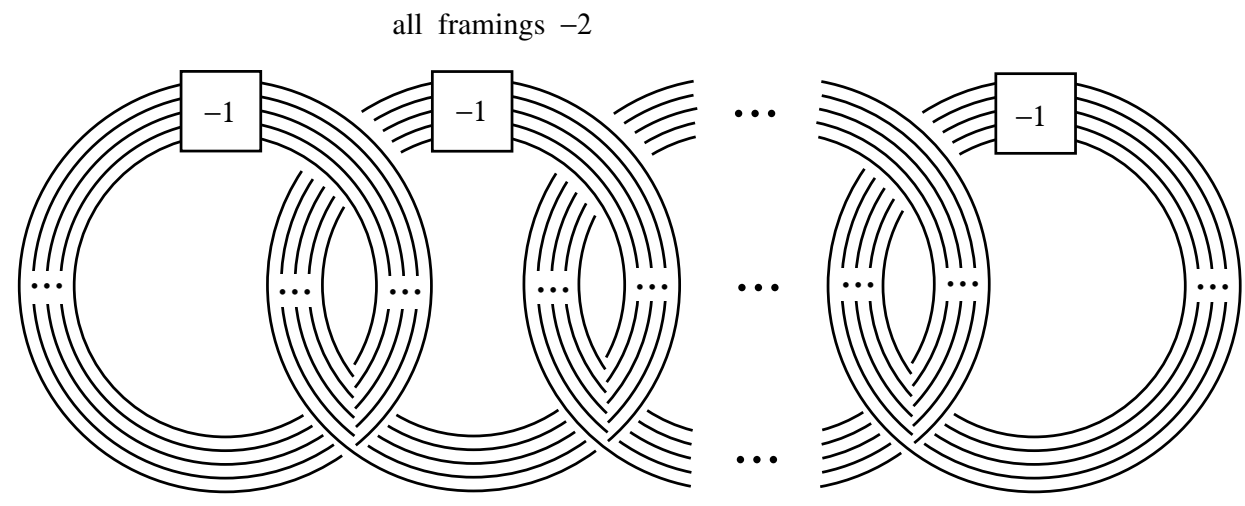

n-1 wheels, $2 n-1$ 2-handles per wheel

\section{Figure 13.}

figure describes a regular neighborhood of the shown configuration of ( $n-$ 1) $(2 n-1) 2$-spheres of square -2 .

We pictorially describe the resolution $V_{r}$ in Figure 14. (We leave the alge-
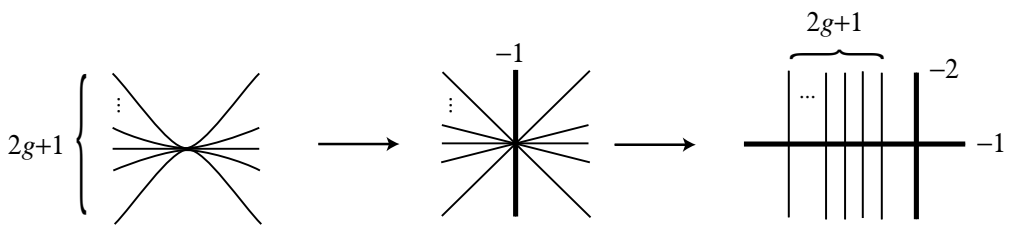

Figure 14 . 
braic computation of the effect of blowing up in local coordinates, such as in [GS], to the reader.) The first blow up shown in Figure 14 alters the singular point of $V$ into $n$ complex lines meeting transversely in a single point; the second blow up then makes the disjoint. Since the union of these lines does not represent an even (i.e., divisible by 2 ) homology class in $H_{2}\left(B^{4} \# 2 \overline{\mathbb{C} P^{2}}\right)$, we cannot form a double cover with it as branch set. Instead, we must recall the fact from the theory of resolutions of complex surface singularities that when forming 2-fold covers branched over resolutions of plane curve singularities, we must include in our branch set any introduced curves of even square $[\mathbf{H K K}]$. Let $S$ be the embedded 2 -sphere of square -2 shown in Figure 14. We may then form the 2-fold cover of $B^{4} \# 2 \overline{\mathbb{C} P^{2}}$ branched over $V_{r} \cup S$. The sphere of square -1 in Figure 14 lifts to this cover as a genus $g$ surface of square -2 . This surface intersects a sphere of square -1 obtained as the lift of the sphere of square -2 in Figure 14. Hence we depict the double cover with the graph in Figure 15. Blowing down the sphere of

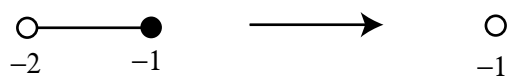

\section{Figure 15.}

square -1 gives a regular neighborhood of a genus $g$ surface of square -1 .

Remark. As this example demonstrates, double covers branched over $V_{d}$ versus $V_{r}$ may not coincide. It is a theorem of Brieskorn that they agree for complex surface singularities which are rational double points. A topological proof of Brieskorn's theorem may be found in [HKK].

A Model Fibration Revisited. Let $\alpha_{1}, \ldots, \alpha_{2 g}$ and $\gamma_{0}$ denote the indicated curves on $\Sigma_{h}$ in Figure 16. Let $N_{0} \rightarrow D^{2}$ denote the genus $h$ Lefschetz fibration of Section 4 with one singular fiber whose vanishing cycle is $\gamma_{0}$. Let $P \rightarrow D^{2}$ denote the Lefschetz fibration with global monodromy given by $\left(\alpha_{1}, \ldots, \alpha_{2 g}\right)^{2(2 g+1)}$. Since

$$
\left(D_{\alpha_{1}} \cdots D_{\alpha_{2 g}}\right)^{2(2 g+1)}=D_{\gamma_{0}}
$$

in $\mathcal{M}_{h}, N_{0}$ and $P$ have the same boundary. Our goal is to compare the branched coverings that produce $N_{0}$ and $P$.

Since all of the curves $\alpha_{1}, \ldots, \alpha_{2 g}$ are nonseparating, $P$ is obtained as a 2 -fold cover of $S^{2} \times D^{2}$ by the methods of Section 3. The ribbon manifold branch set $B$ can be explicitly drawn in the boundary, as in Figure 17. 
genus $g$

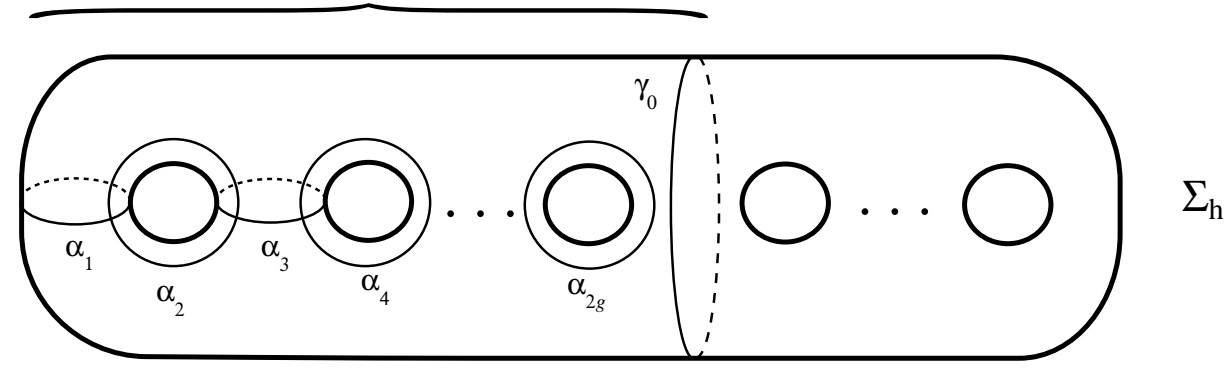

Figure 16.

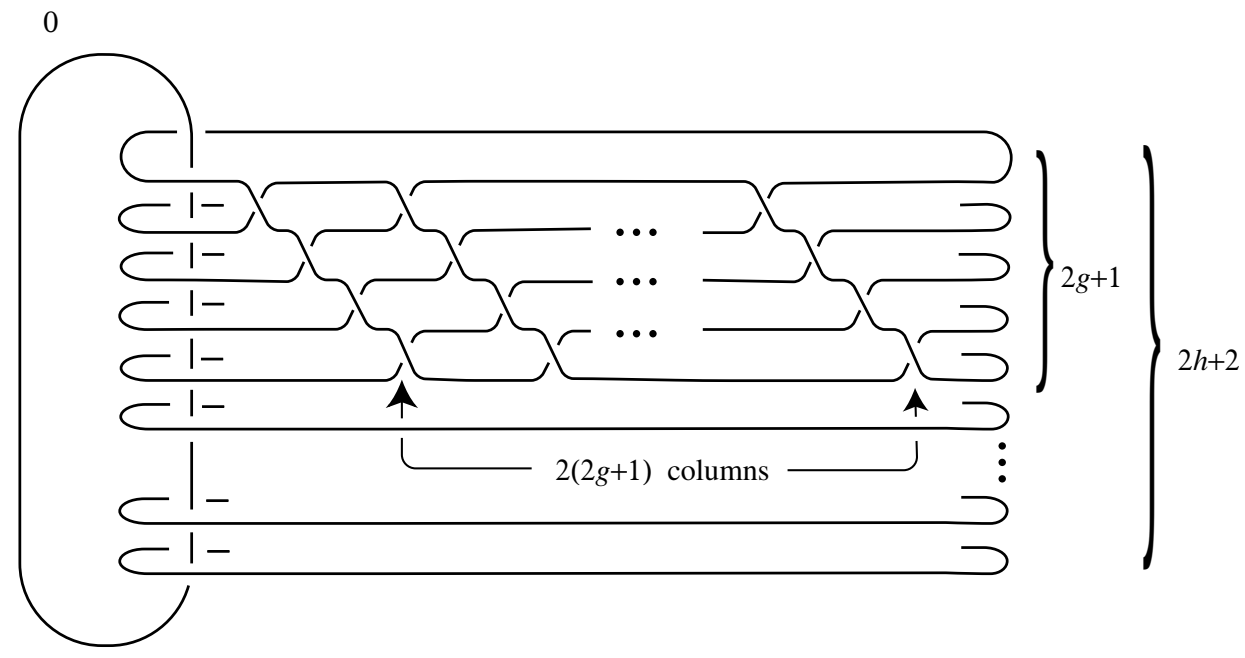

Figure 17.

Let $D$ denote the 3-ball in $\partial\left(S^{2} \times D^{2}\right)$ shown in Figure 18, and let $E=D \times$ $I$ denote a 4-ball collar neighborhood of $D$. Then $B \cap E$ is the fibered Seifert surface of minimal genus for a $(2 g+1,2(2 g+1))$ torus link, so $(E, B \cap E) \cong$ $\left(B^{4}, V_{d}\right)$, where $V_{d}$ is the deformation of an infinitely close $n$-tuple point, with $n=2 g+1$. Hence we may delete this 4 -ball, and replace it with $\left(B^{4}, V\right)$, replacing the deformation $V_{d}$ with $V$. This produces an immersed surface $C=\left(B-V_{d}\right) \cup V$. Alternatively, we may delete $\left(B^{4}, V_{d}\right)$, and replace it with $\left(B^{4} \# 2 \overline{\mathbb{C} P^{2}}, V_{r}\right)$. (It is a routine Kirby calculus exercise to verify that the boundary diffeomorphism $\partial\left(B^{4} \# 2 \overline{\mathbb{C} P^{2}}\right) \rightarrow S^{3}$ given by blowing down the final picture in Figure 14 sends $\partial\left(V_{r}\right)$ to a $(2 g+1,2(2 g+1))$ torus link. Thus we may match up $\partial\left(V_{r}\right)$ and $\partial\left(V_{d}\right)$.) Doing this transforms the manifold in 


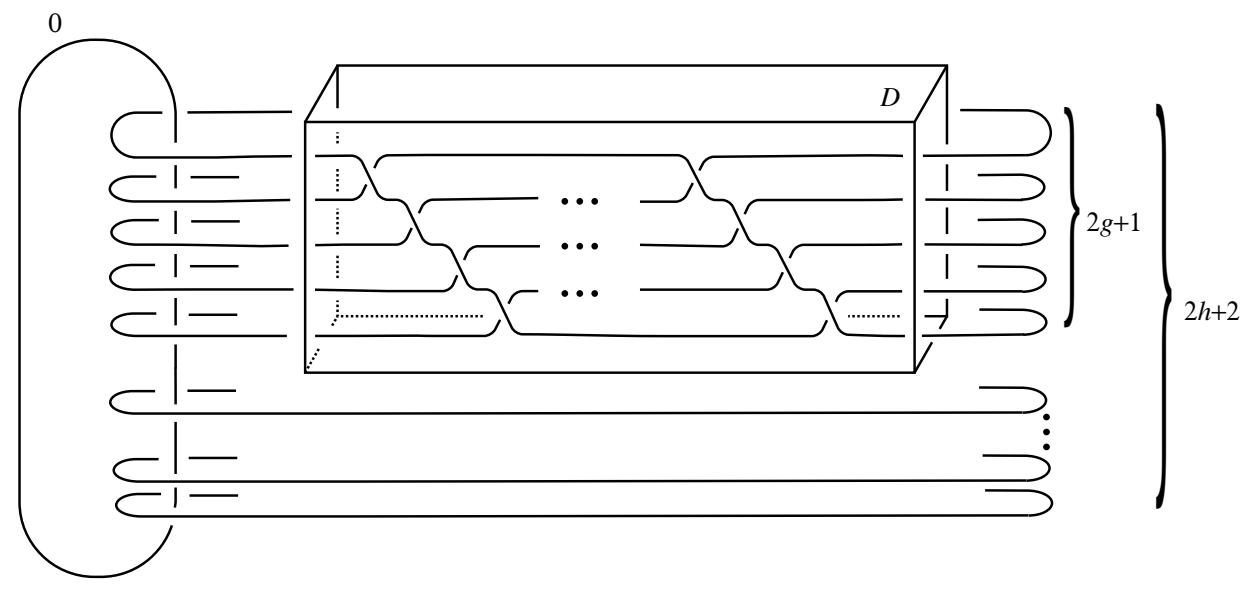

Figure 18.

Figure 18 to the manifold in Figure 6. By Proposition 6, forming the 2-fold branched cover of the latter branched over $B^{\prime}=\left(B-V_{d}\right) \cup V_{r} \cup S$ produces a non-relatively minimal Lefschetz fibration whose relative minimalization is $N_{0} \rightarrow D^{2}$. We have shown the following.

Proposition 9. (a) The branch surface for the branched covering $N_{0} \# \overline{\mathbb{C} P^{2}} \rightarrow S^{2} \times D^{2} \# 2 \overline{\mathbb{C} P^{2}}$ is the resolution of the immersed surface $C \cup S$ in $S^{2} \times D^{2}$.

(b) The branch surface for the branched covering $P \rightarrow S^{2} \times D^{2}$ is the deformation of the immersed surface $C$ in $S^{2} \times D^{2}$.

The proof of Theorem 8 used the action of $\mathcal{H}_{h}$ on separating curves to transfer the model of a branched cover giving $\gamma_{0}$ as a vanishing cycle to give any symmetric separating curve as a vanishing cycle. Similar arguments give the following.

Corollary 10. Let $M \rightarrow S^{2}$ be a relatively minimal hyperelliptic genus $h$ Lefschetz fibration whose monodromy includes $\sigma$ separating vanishing cycles. Then the branch surface for the branched covering $M \# \sigma \overline{\mathbb{C} P^{2}} \rightarrow \mathbb{C} P^{2} \#(2 \sigma+$ $1) \overline{\mathbb{C} P^{2}}$ is the resolution of an immersed surface in $S^{2} \times S^{2}$ or $S^{2} \widetilde{\times} S^{2}$ with only infinitely close $n$-tuple point singularities. 
Proof. If $\gamma_{i}$ is any separating vanishing cycle which bounds a genus $g$ surface, then we have as in the proof of Theorem 8 a commutative diagram

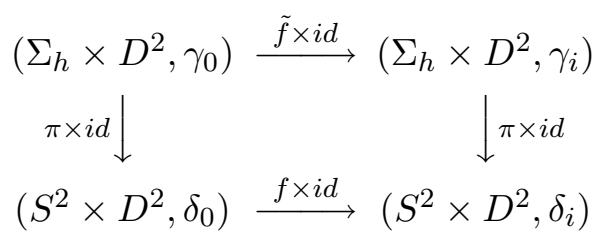

which extends to a commutative diagram

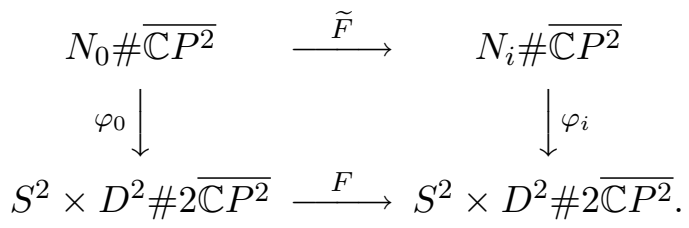

The portion of the branch set constructed in the proof of Theorem 8 to produce $\gamma_{i}$ as a separating vanishing cycle can be described as the result of replacing $(f \times i d)\left(B^{4}, V\right)$ with $F\left(B^{4} \# 2 \overline{\mathbb{C} P^{2}}, V_{r}\right)$.

Remark. In general, a right-handed Dehn twist about a separating curve on $\Sigma_{h}$ can always be expressed as a product of right-handed Dehn twists about nonseparating curves. Thus, we can always consider the operation on Lefschetz fibrations of removing the neighborhood of a singular fiber with a separating vanishing cycle, and replacing it with a fibration given by the appropriate collection of nonseparating vanishing cycles (and vice versa).

We can isolate this operation to smaller neighborhoods, as follows. When these fibrations are hyperelliptic and viewed as branched covers, we have shown that this operation can be phrased as replacing a submanifold of the base diffeomorphic to $\left(B^{4} \# 2 \overline{\mathbb{C} P^{2}}, V_{r}\right)$ with $\left(B^{4}, V_{d}\right)$, and forming 2-fold covers. Lifting these submanifolds to the branched cover, these operations involve trading back and forth (after blowing down exceptional curves) the regular neighborhood of an embedded genus $g$ surface of square -1 as in Figure 15 with the regular neighborhood of a configuration of embedded 2-spheres as in Figure 13.

\section{References}

[AK] S. Akbulut and R. Kirby, Branched covers of surfaces in 4-manifolds, Math. Ann., 252 (1980), 111-131.

[BPV] W. Barth, C. Peters and A. Van de Ven, Compact Complex Surfaces, Ergebnisse der Mathematik, Springer-Verlag, Berlin, 1984.

[B] J. Birman, Braids, links, and mapping class groups, Ann. of Math. Stud., 82, Princeton Univ. Press, Princeton, NJ, 1975. 
[BH] J. Birman and H. Hilden, On the mapping class groups of closed surfaces as covering spaces, Advances in the theory of Riemann surfaces, Ann. of Math. Stud., 66, Princeton Univ. Press, Princeton, NJ, (1971), 81-115.

[D] S. Donaldson, Lefschetz fibrations in symplectic geometry, Doc. Math. J. DMV Extra Volume ICM, (1998) II, 309-314.

[GS] R. Gompf and A. Stipsicz, An introduction to 4-manifolds and Kirby calculus, book to appear.

[HKK] J. Harer, A. Kas and R. Kirby, Handlebody decompositions of complex surfaces, Mem. Amer. Math. Soc., 62 (1986), number 350.

[K] A. Kas, On the handlebody decomposition associated to a Lefschetz fibration, Pacific J. Math., 89 (1980), 89-104.

[Ma] Y. Matsumoto, Lefschetz fibrations of genus two - a topological approach, Proceedings of the 37th Taniguchi Symposium on Topology and Teichmüller Spaces, ed. Sadayoshi Kojimaet al., World Scientific, (1996), 123-148.

[Mi] J. Milnor, Singular points of complex hypersurfaces, Ann. of Math. Stud., 61, Princeton Univ. Press, Princeton, NJ, 1968.

[Ms] B. Moishezon, Complex surfaces and connected sums of complex projective planes, Lecture Notes in Math., 603, Springer, New York, 1977.

[Mo] J.M. Montesinos, 4-manifolds, 3-fold coverings, and ribbons, Trans. Amer. Math. Soc., 245 (1978), 453-467.

[P1] U. Persson, Chern invariants of surfaces of general type, Compositio Math., 43 (1981), 3-58.

[P2] - Genus 2 fibrations revisited, Lecture Notes in Math., 1137, Springer, New York, (1992), 133-144.

[ST] B. Siebert and G. Tian, On hyperelliptic $C^{\infty}$-Lefschetz fibrations of four-manifolds, preprint math.GT/9903006.

[S] I. Smith, Symplectic geometry of Lefschetz fibrations, Dissertation, Oxford University, 1998.

Received February 1, 1999. The author was supported by NSF grant DMS 97-29992.

Department of Mathematics

California State University, Northridge

Northridge, CA 91330

E-mail address: terry.fuller@csun.edu 